\title{
System non-linearities induced by skyhook dampers
}

\author{
Mehdi Ahmadian, Brian Reichert and \\ Xubin Song \\ Advanced Vehicle Dynamics Laboratory, Department \\ of Mechanical Engineering, MC - 0238, Virginia \\ Tech, Blacksburg, Virginia 24061, USA \\ Tel.: +1 540231 4920; Fax: +1 540231 9100; \\ E-mail:ahmadian@vt.edu
}

\begin{abstract}
The nonlinearities induced by skyhook dampers are studied experimentally and analytically using a single degree of freedom base-excited system that is representative of the systems that such dampers are often used in. The experimental results are used to show that the nonlinearities introduced by skyhook dampers manifest themselves as high frequency peaks in the frequency spectrum of the system response. More precisely, using a pure tone input with a known frequency, it is shown that these peaks occur at odd multiples of the system input (or driving) frequency. Using a Fourier series analysis, it is proven that the nonlinearities are caused by the switching policy of skyhook dampers, in which the damper force is changed according to the relative sign of the sprung mass absolute velocity and the relative velocity across the damper. The analysis shows that the skyhook damper force always contains a frequency component that is equal to the frequency of the system input (as is expected for a linear system), in addition to other frequencies that are odd multiples of the input frequency. The damping force peak that occurs at the input frequency is necessary for controlling the forced response of the system. The peaks at higher frequencies, however, are not desirable - although always present - because they introduce corresponding peaks in the system response that can cause ancillary vibration problems. For most systems, the high frequency peaks can significantly diminish any isolation benefits that are gained by the skyhook damper. When using skyhook dampers, such effects must be considered and their impact on the system dynamics studied carefully.
\end{abstract}

\section{Introduction}

The idea of skyhook dampers has been in existence for more than two decades. Introduced by Crosby and Karnopp [8], skyhook dampers, which have been stud- ied most often for vehicle suspensions, draw a small amount of energy to operate a valve that adjusts the damping force. The damper valve can be a mechanical element (as is the case for mechanically-adjustable dampers) or a fluid valve that takes advantage of the rheological changes of the damper fluid, such as magneto rheological dampers $[4,5]$.

The virtues of skyhook dampers versus conventional, passive dampers have been addressed in many past studies $[1-3,6,7,13,14]$. Using various analytical methods, these studies have concluded that in nearly all cases semiactive dampers can reduce vibration transmission across the suspension and better control the suspended (or sprung) body, as compared to passive dampers. Further, these studies have led to a number of variations to skyhook control, which are reviewed by Ivers and Miller [11].

Although there exists a large number of studies that provide a comprehensive analysis of skyhook control method and its variations, to the best of our knowledge none of these studies have examined the nonlinearities that are induced by the skyhook dampers. Even in studies where such a phenomenon may have occurred, it has been dismissed as the existence of noise in the system.

In this study, we intend to provide a comprehensive analysis of the system nonlinearities that are induced by skyhook dampers. After providing an overview of skyhook control and the test setup that is used for generating our results, we describe the nonlinearities that are introduced into the system response by skyhook dampers. Further, we will provide a mathematical explanation of the nonlinearities and how they relate to the system input.

\section{Background}

Using the single degree of freedom base-excited system shown in Fig. 1, the equations governing skyhook control can be described by: 


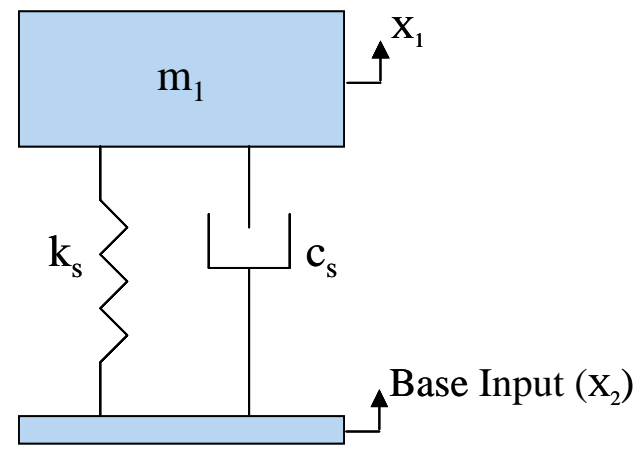

Fig. 1. Single Degree of Freedom (SDOF) base excited system.

$$
\left\{\begin{array}{l}
V_{1} V_{12}>0 F_{s a}=G_{s} V_{1} \\
V_{1} V_{12}<0 F_{s a}=0
\end{array}\right.
$$

where

$V_{1}=$ absolute velocity of the sprung mass,

$V_{12}=$ relative velocity across the suspension,

$F_{s a}=$ skyhook damping force, and

$G_{s}=$ a gain factor that is commonly used such that the full damping range of the skyhook damper can be used.

The logic of the skyhook control policy is as follows. When the relative velocity of the damper is positive, the force of the damper pulls down on the vehicle body; when the relative velocity is negative, the force of the damper pushes up on the body. Thus, when the absolute velocity of the vehicle body is negative, it is traveling downwards and the maximum value of damping is desired to push up on the body, while the minimum value of damping is desired to continue pulling down on the body. If, however, the absolute velocity of the body is positive and it is traveling upward, the maximum value of damping is desired to pull down the body, while the minimum value of damping is desired to further push the body upward. The skyhook semiactive policy emulates the ideal body displacement control configuration of a passive damper "hooked" between the body mass and the "sky", as depicted in Fig. 2; hence, the name "skyhook".

\section{Problem statement}

The issue that is discussed in this paper became apparent during a series of tests that the authors conducted on the suspension system shown in Fig. 3. During these tests, it was observed that when the system contains a skyhook damper and is excited at the base with the input shown in Fig. 4, the accelerations that are measured on the sprung mass (i.e., the system response) are as

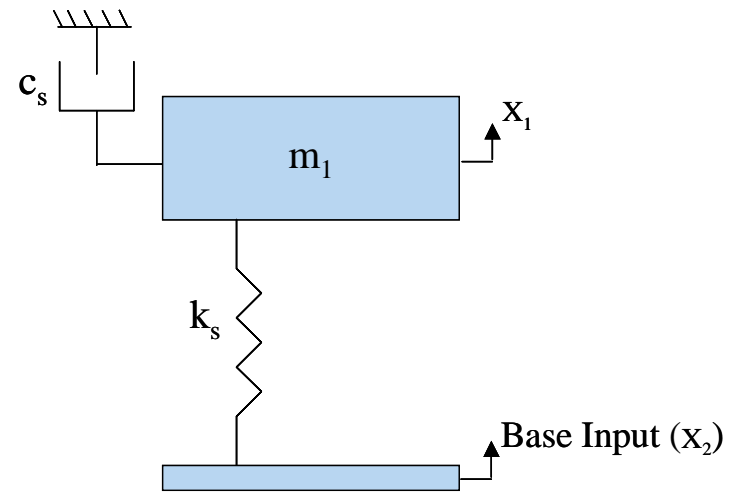

Fig. 2. Schematics of the idealized skyhook damper.

shown in Fig. 5. Figure 5 shows that in addition to the main peak that is directly due to the input, a secondary peak is evident in the Power Spectral Density (PSD) of the sprung mass acceleration, which was not expected. Since the natural frequency of the sprung mass (occurring at approximately $1.4 \mathrm{~Hz}$ ) is far below the second peak and the suspension elements are linear within the test range, it was fully anticipated that the system response contains only a single peak, coinciding with the input peak. This study will provide a mathematical and experimental analysis of the source of the second peak and the effect that it can have on systems with skyhook dampers.

\section{Test setup}

The details of the test rig that was used for this study, shown in Fig. 3, can be found in Reichert [15] and Song [16]. The test rig closely resembles the system in Fig. 1, where the base is excited by a hydraulic actuator with a maximum force capacity of $2000 \mathrm{lb}$. The sprung mass is supported on a suspension that consists of an airspring and an adjustable magneto rheological (MR) damper. The airspring has a linear spring constant within the range of the relative displacement that was used in our tests. The MR damper force characteristics are bilinear, as shown for a typical MR damper in Fig. 6. As Fig. 6 indicates, the damper force is directly proportional to the current supplied to the damper. An MR damper was used over a mechanically adjustable damper because of its availability, ease of use, and the growing trend of using such dampers for vehicle applications.

A standard input profile known as ISO Class 2 Excitation (also known as "ISO 2 Excitation"), shown in Fig. 4, was used for exciting the system [10]. The ab- 


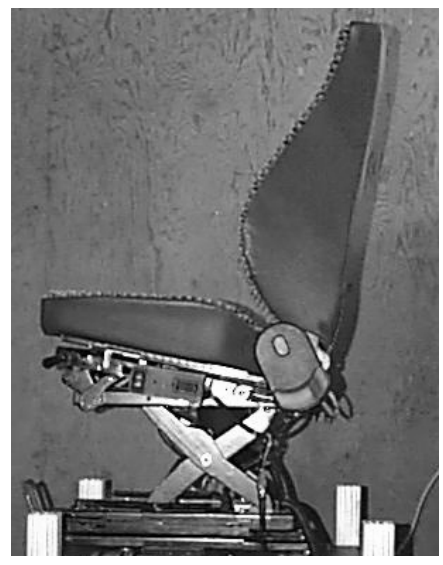

(a)

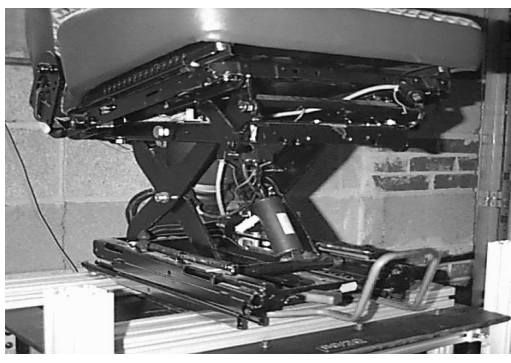

(b)

Fig. 3. Base-Excited Single Degree of Freedom System (SDOF) test setup.

solute velocity needed for skyhook control was derived from an accelerometer on the sprung mass, and the relative velocity across the suspension was measured directly using a Linear Voltage Differential Transformer (LVDT). A dSPACE Autobox was used to implement the skyhook control and acquire the data needed for our study.

\section{Results}

Using the system in Fig. 3, we start our analysis by evaluating the response of the system with different suspensions to a pure tone input with a frequency of 1.4 Hz. The suspensions that are considered include:

1. A passive damper with large damping ratio (referred to as "stiff damper"),

2. A passive damper with small damping ratio (referred to as "soft damper"),

3. A semiactive skyhook damper, controlled according to Eq. (1).

The response of the system, as indicated by the PSD of the sprung mass acceleration, with stiff damper indicates a single peak at $1.4 \mathrm{~Hz}$, coinciding with the input frequency, as shown in Fig. 7. Of course, this is fully expected because it is well known that a linear, singledegree-of-freedom, base-excited system (such as a test system) will have a response that has the same frequency as the input frequency, with a phase shift that depends on the damping ratio of the suspension [9]. The results with the soft damper, shown in Fig. 8, closely resemble Fig. 7, in the sense that a single peak occurs at the same frequency as the input peak.
When the damper force is controlled according to the skyhook control in Eq. (1), the system response exhibits other peaks in addition to the $1.4 \mathrm{~Hz}$ peak. The PSD of the sprung mass acceleration, included in Fig. 9, shows peaks at 4.2, 7.0, 9.8, 12.6, 15.4, 18.2 . . Hz, all odd multiples of the input frequency. We will refer to these peaks, collectively, as "secondary peaks". This behavior is the same as what was described in Fig. 4, when the system was excited by the ISO 2 input.

To mathematically evaluate the source of the secondary peaks, we will first examine the phase relationship between the absolute velocity $\left(V_{1}\right)$ and the relative velocity $\left(V_{12}\right)$. Recall from our earlier discussions that the transmissibility for the ideal skyhook is

$$
\frac{X_{1}}{X_{2}}=\frac{1}{1-\left(\frac{\omega}{\omega_{n}}\right)^{2}+j 2 \zeta_{S K Y}\left(\frac{\omega}{\omega_{n}}\right)}
$$

where $\zeta_{S K Y}$ is the skyhook damping ratio. Equation (2) can be used to derive the transfer function between the relative displacement across the suspension and the base input as

$$
\begin{aligned}
\frac{X_{1}-X_{2}}{X_{2}} & =\frac{X_{12}}{X_{2}} \\
& =\frac{\left(\frac{\omega}{\omega_{n}}\right)^{2}-j 2 \zeta_{S K Y}\left(\frac{\omega}{\omega_{n}}\right)}{1-\left(\frac{\omega}{\omega_{n}}\right)^{2}+j 2 \zeta_{S K Y}\left(\frac{\omega}{\omega_{n}}\right)}
\end{aligned}
$$

The phase difference between the two transmissibilities in Eqs (2) and (3) will only be affected by the numerator since the denominators are identical. Inspecting the phase of the numerators relative to one another indicates that the absolute velocity $\left(V_{1}\right)$ has a phase 


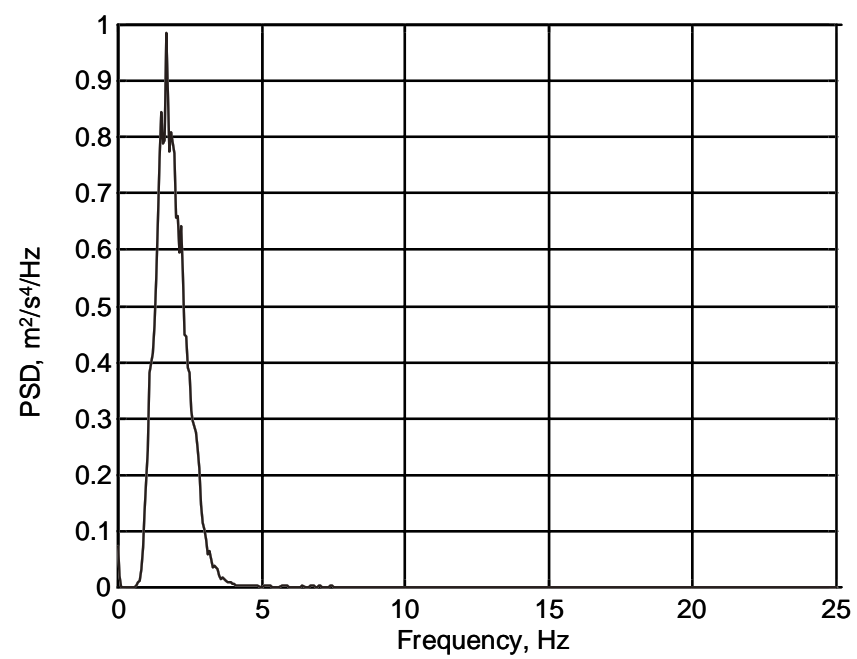

(a)

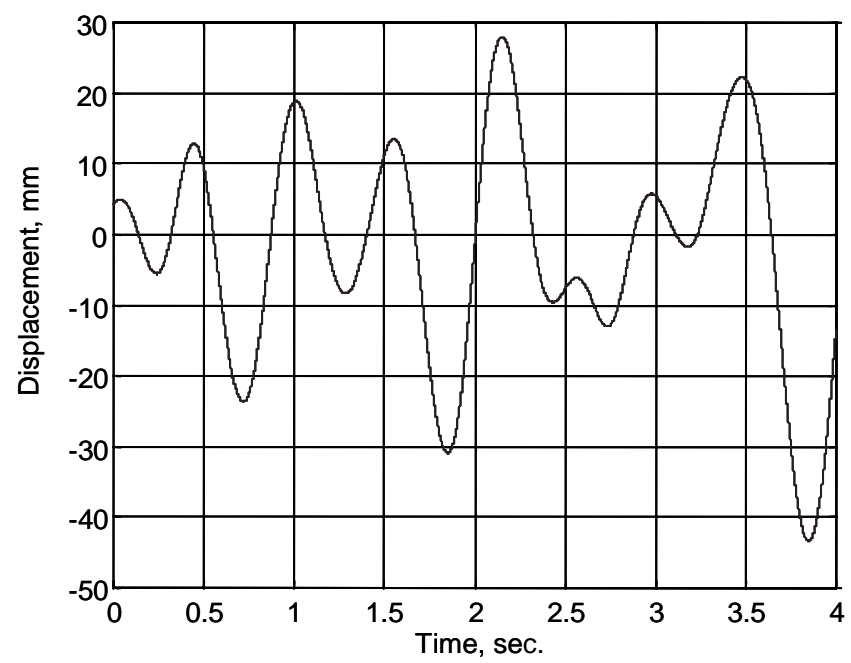

(b)

Fig. 4. ISO Class 2 (ISO 2) excitation; (a) Power spectral density; (b) Sample time trace.

lead with respect to the relative velocity $\left(V_{12}\right)$, for all frequencies.

Now, let us examine the current that is supplied to the skyhook damper due to a pure tone $1.4 \mathrm{~Hz}$ input. For a magneto rheological damper, such as the damper used in this study, the damper current is directly proportional to the damper force. Figure 10, which shows the PSD of the damper current, indicates that the first frequency component is at $2.8 \mathrm{~Hz}$, or twice the excitation frequency. The reason for this phenomenon can best be described by evaluating the phase relationship between $V_{1}$ and $V_{12}$, as shown in Fig. 11. This figure shows the relative velocity $\left(V_{12}\right)$, absolute velocity $\left(V_{1}\right)$, and damper current for a pure tone excitation near the resonant frequency of the suspension. Since the absolute and relative velocities are out of phase, they will have the same sign twice in each cycle. This implies that the damper force, and therefore the damper current, complete two cycles for every cycle of the driving force; hence, causing the peak at $2.8 \mathrm{~Hz}$, twice the driving frequency of the system. Although Fig. 11 shows the current for an on-off semiactive damper, the same concept applies to a continuously variable damper. For on-off skyhook damper, the damper force is adjusted to only two values, the minimum and maximum force attainable by the damper. This is in contrast to a continuously adjustable damper, in which the damping force is directly proportional to the absolute velocity $\left(V_{1}\right)$, as 


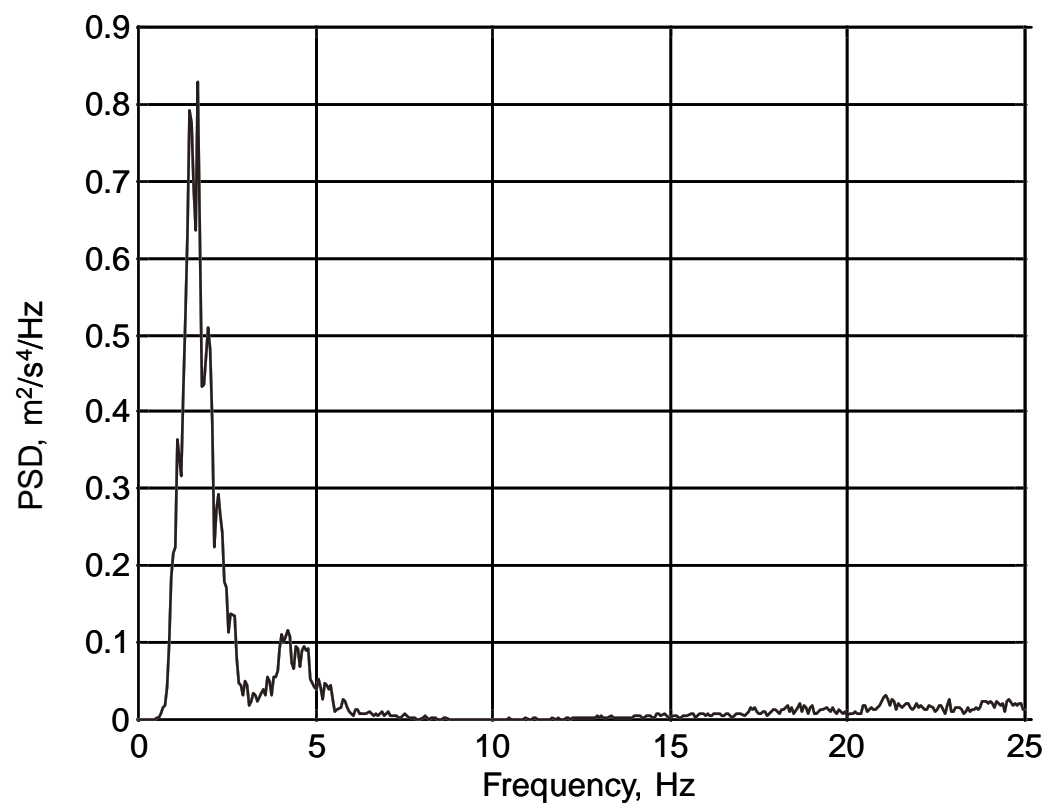

Fig. 5. Sprung mass acceleration (system response) power spectral density due to ISO 2 excitation for a skyhook damper.

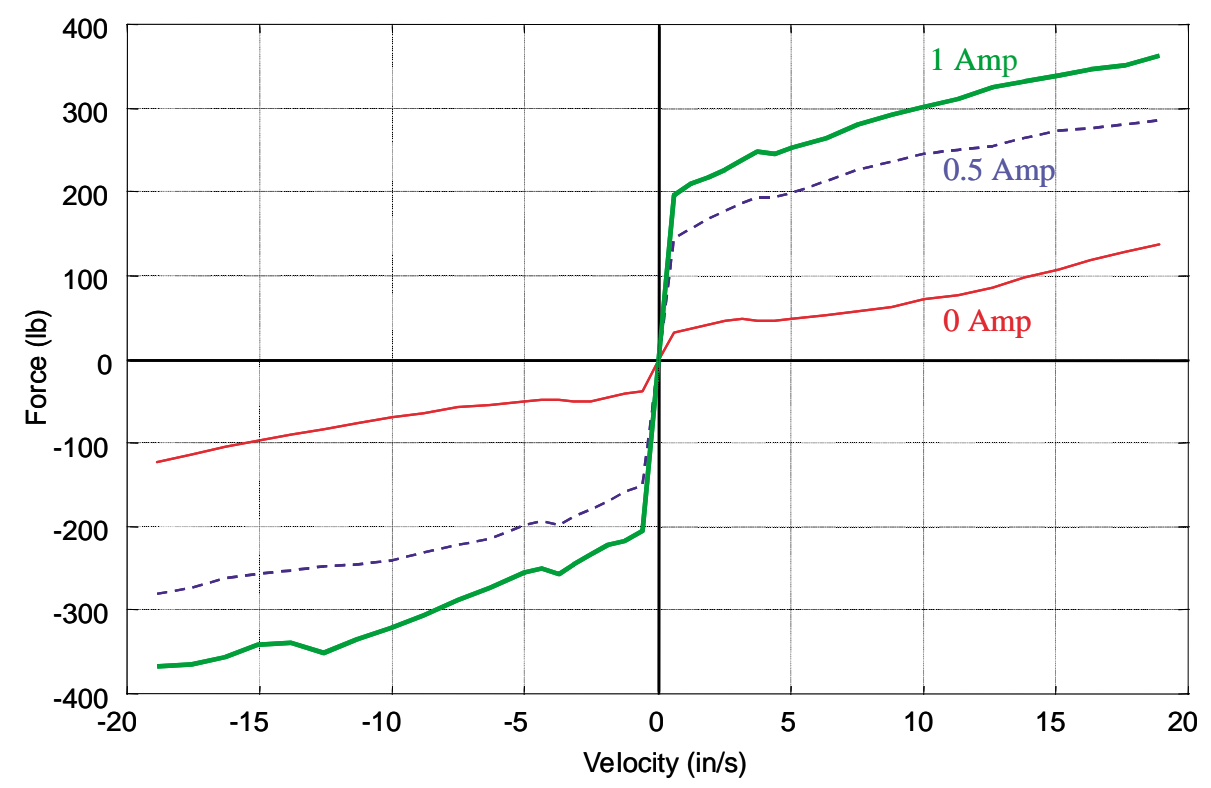

Fig. 6. Typical magneto rheological damper force characteristics for different currents.

described in Eq. (1) and shown in Fig. 12.

If $\frac{\phi}{\omega}$ is defined as the time duration that the damper is on, the absolute velocity $\left(V_{1}\right)$ in Fig. 12 can be expressed as

$$
\begin{aligned}
& V_{1}(t)=V_{1} \sin [\omega t+(\pi-\phi)] \\
& V_{1}(t)=V_{1} \sin [\omega t+\lambda]
\end{aligned}
$$

$$
\lambda=\pi-\phi
$$

Using Eqs (1) and (4)-(6), the damper force $F_{s a}(t)$ can be mathematically defined over one period by

$$
\begin{cases}F_{s a}=G_{s} V_{1} \sin (\omega t+\lambda) & 0 \leqslant t<\frac{\phi}{\omega} \\ F_{s a}=0 & \frac{\phi}{\omega} \leqslant t<\frac{\pi}{\omega} \\ F_{s a}=G_{s} V_{1} \sin (\omega t+\lambda) & \frac{\phi}{\omega} \leqslant t<\frac{\pi+\phi}{\omega} \\ F_{s a} & \frac{\pi+\phi}{\omega} \leqslant t<\frac{2 \pi}{\omega}\end{cases}
$$




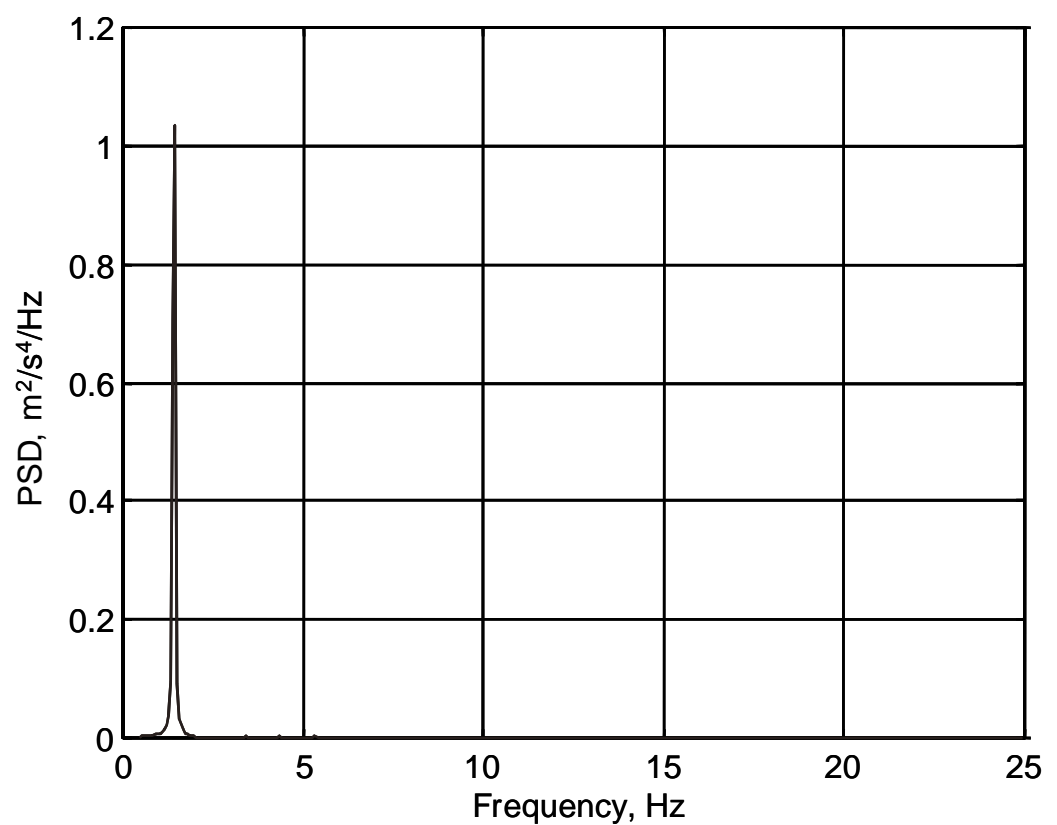

Fig. 7. Sprung mass acceleration (system response) power spectral density due to a $1.4 \mathrm{~Hz}$ pure tone excitation for a hard damper.

The Fourier series expansion of Eq. (7) yields

$$
\begin{aligned}
& F_{s a}(t) \\
= & \frac{a_{0}}{2}+\sum_{n=1}^{\infty}\left(a_{n} \cos n \omega t+b_{n} \sin n \omega t\right)
\end{aligned}
$$

where

$$
\begin{aligned}
a_{0}= & 0 \\
a_{n}= & \frac{G_{s} V_{1}}{\pi}\left\{\operatorname { c o s } \lambda \left[\frac{-\cos (1+n) \phi}{2(1+n)}\right.\right. \\
& \left(1+(-1)^{n+1}\right) \\
& -\frac{\cos (1-n) \phi}{2(1-n)}\left(1+(-1)^{n+1}\right) \\
& +\frac{1}{2(1+n)}\left(1+(-1)^{n+1}\right) \\
& \left.+\frac{1}{2(1-n)}\left(1+(-1)^{n+1}\right)\right] \\
& +\sin \lambda\left[\frac{\sin (1-n) \phi}{2(1-n)}\left(1+(-1)^{n+1}\right)\right. \\
& \left.\left.+\frac{\sin (1+n) \phi}{2(1+n)}\left(1+(-1)^{n+1}\right)\right]\right\} \\
b_{n}= & \frac{G_{s} V_{1}}{\pi}\left\{\operatorname { s i n } \lambda \left[\frac{-\cos (1+n) \phi}{2(1+n)}\right.\right. \\
& \left(1+(-1)^{n+1}\right)
\end{aligned}
$$

$$
\begin{aligned}
& -\frac{\cos (1-n) \phi}{2(1-n)}\left(1+(-1)^{n+1}\right) \\
& +\frac{1}{2(1-n)}\left(1+(-1)^{n+1}\right) \\
& \left.+\frac{1}{2(1+n)}\left(1+(-1)^{n+1}\right)\right] \\
& +\cos \lambda\left[\frac{\sin (1-n) \phi}{2(1-n)}\left(1+(-1)^{n+1}\right)\right. \\
& \left.\left.-\frac{\sin (1+n) \phi}{2(1+n)}\left(1+(-1)^{n+1}\right)\right]\right\}
\end{aligned}
$$

For even values of $n$, the Fourier coefficients $a_{n}$ and $b_{n}$ will be zero. Therefore, the damper force exists only for the odd values of $n$.

According to Eqs (8) and (9), the skyhook damping force resulting from a pure tone input with a frequency of $\omega$ has multiple frequencies. The first frequency occurs at the input (or driving) frequency, which is necessary for controlling the forced response of the system. The other frequencies occur at odd multiples of the input frequency and are not necessarily desirable, although they always exist, because they introduce secondary peaks in the system response that can cause ancillary vibration problems, as shown in Fig. 9. The most noticeable frequency in the damping force, besides the input frequency, will be at three times the input frequency because of the nature of the Fourier 


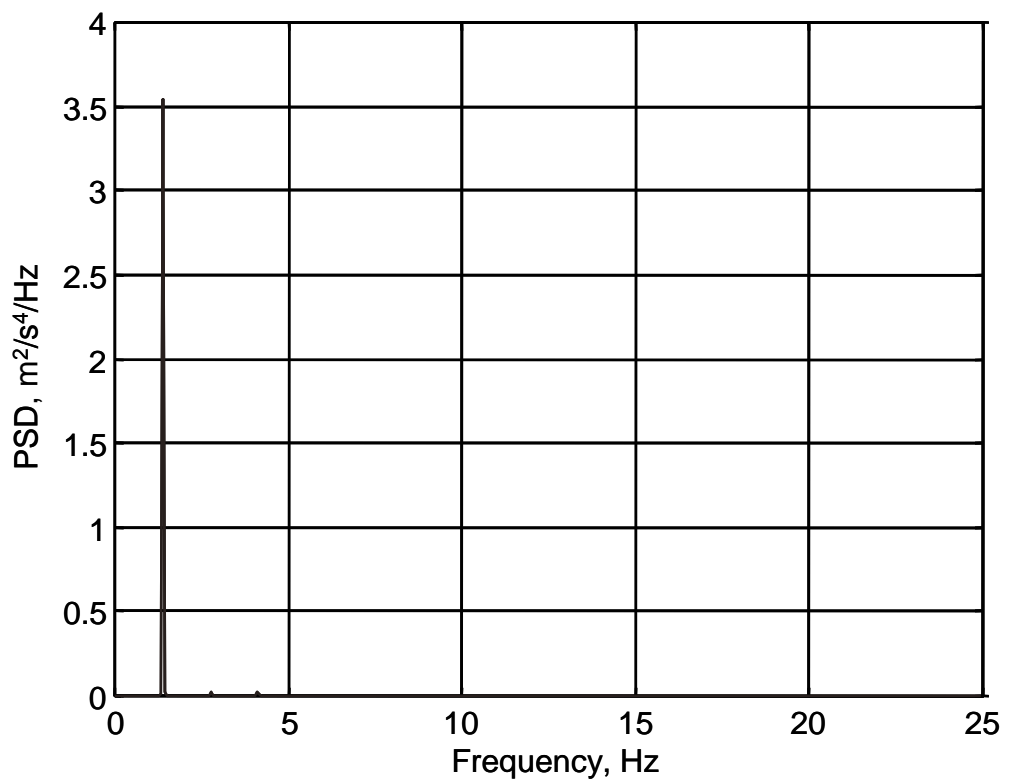

Fig. 8. Sprung mass acceleration (system response) power spectral density due to a $1.4 \mathrm{~Hz}$ pure tone excitation for a soft damper.

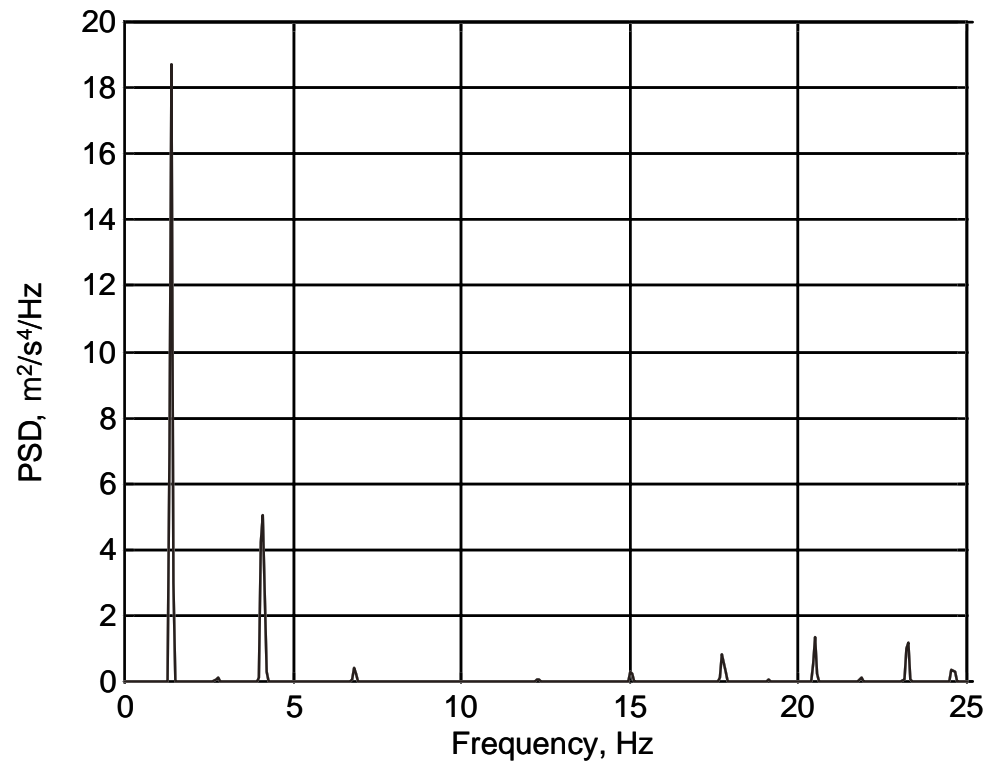

Fig. 9. Sprung mass acceleration (system response) power spectral density due to a $1.4 \mathrm{~Hz}$ pure tone excitation for a skyhook damper.

series, in which the magnitude for higher frequencies decay toward zero.

\section{Summary}

The nonlinearities induced by skyhook dampers were studied experimentally and analytically for a single degree of freedom system, representative of the sys- tems that such dampers are often used in. Through experimental results, it was shown that the nonlinearities introduced by skyhook dampers appear as a series of peaks in the power spectral density (PSD) of the system response, at frequencies higher than the system input frequency. Using a $1.4 \mathrm{~Hz}$ pure tone base input, it was further shown that these higher frequencies are odd multiples of the input frequency. For an on- 


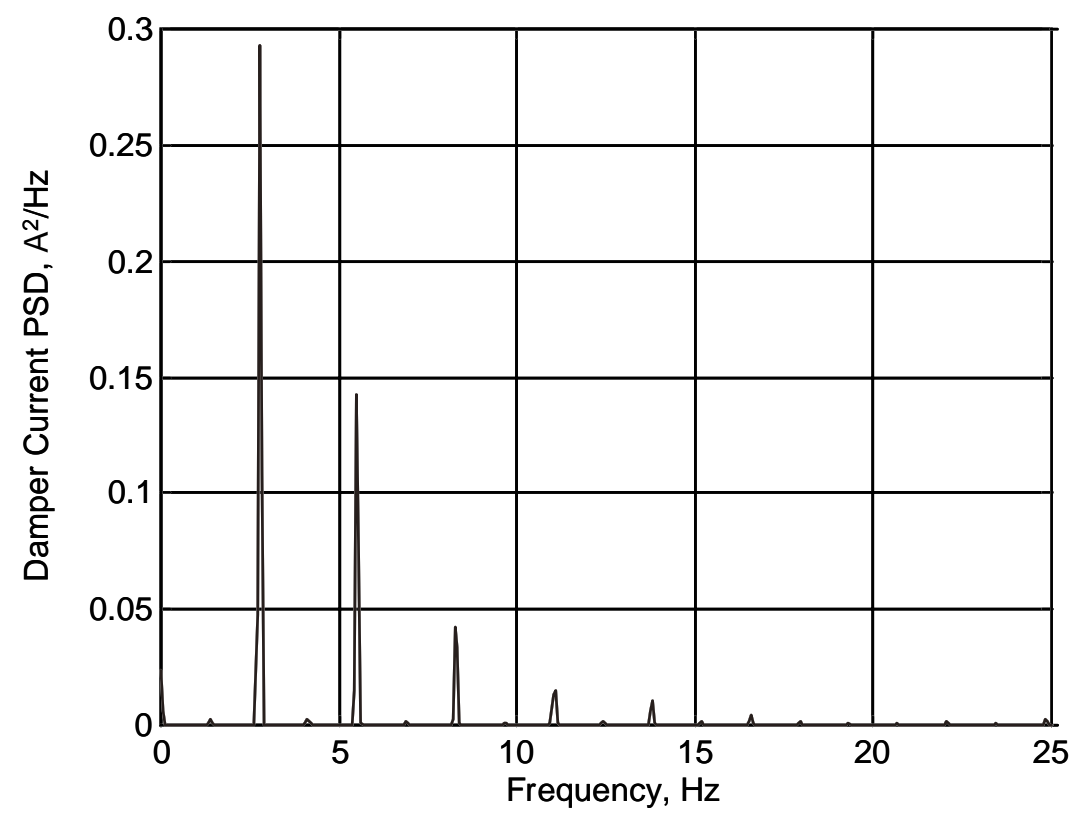

Fig. 10. Damper current power spectral density due to a $1.4 \mathrm{~Hz}$ pure tone excitation for a skyhook damper.

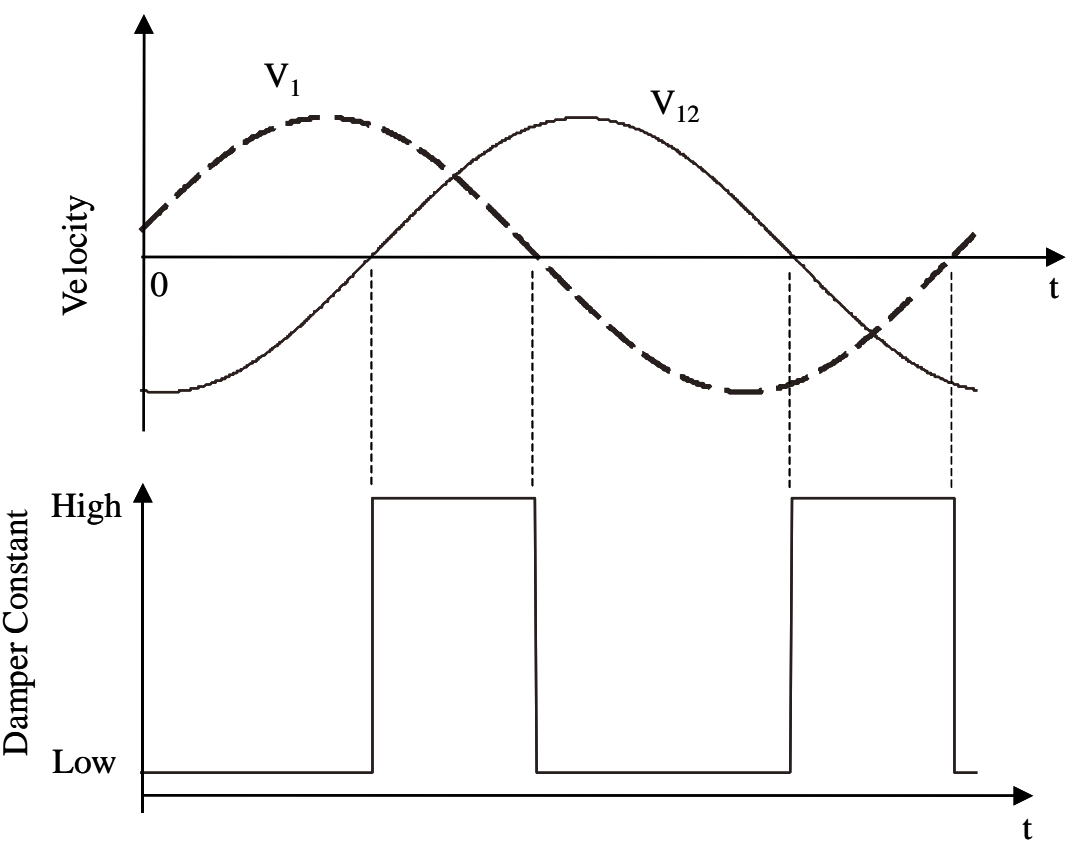

Fig. 11. Relationship between $V_{1}, V_{12}$, and damper current due to a pure tone excitation for an on-off skyhook damper.

off damper, it was determined that the damping constant (or damper current, in case of magneto rheological dampers) switches between the low and high state at twice the frequency of the driving frequency, due to the phase shift that always exists between the absolute velocity of the sprung mass and the relative velocity across the damper. Extending this concept to a continuously variable skyhook damper, it was shown that there exits two zero crossing of the absolute velocity during each relative velocity cycle. This gives rise to two cycles of the damper force for each cycle of the relative velocity. In each of its cycles, the damper force 

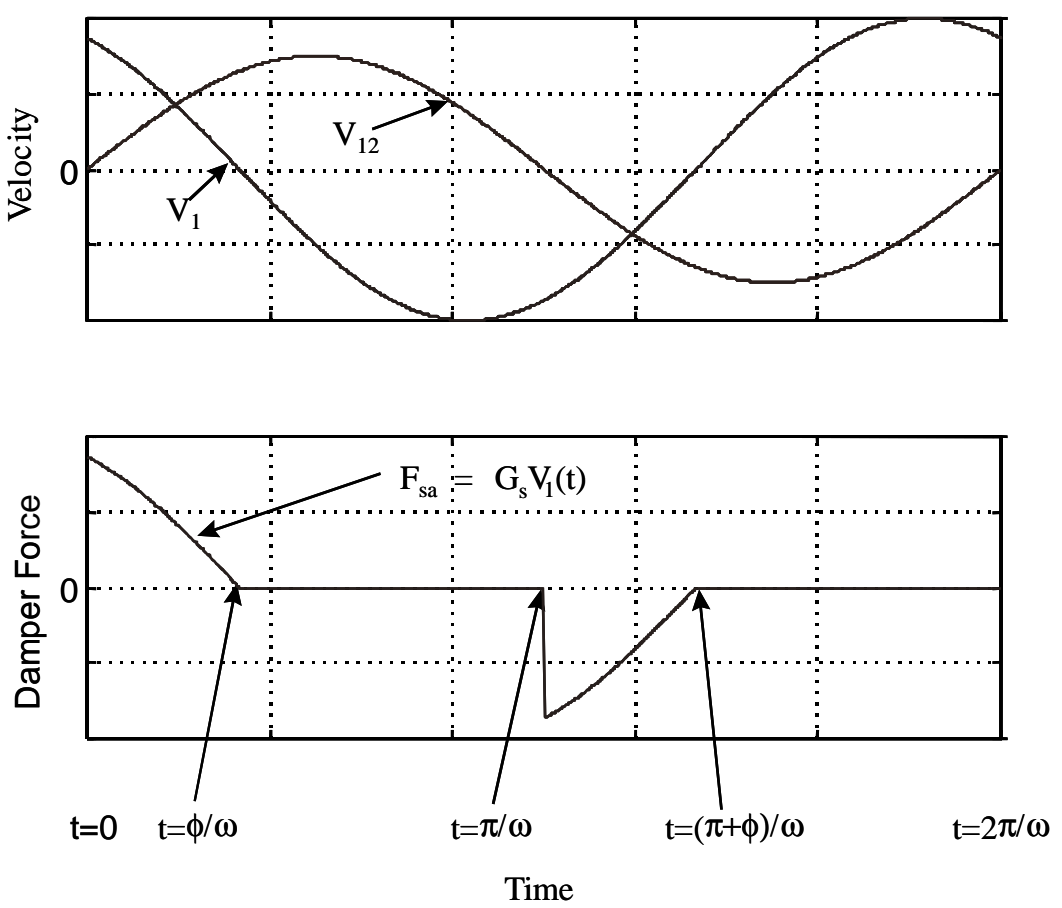

Fig. 12. Relationship between $V_{1}, V_{12}$, and damper current due to a pure tone excitation for a continuously variable skyhook damper.

switches between the off and on state once, and in the on state it is proportional to the absolute velocity of the sprung mass. Using Fourier series, it was shown that such a damping force contains multiple frequencies that include the input (driving) frequency and its odd multiples. It was further shown that the amplitude of the peaks at the odd multiples of the driving frequency decreases as the frequency for each peak increases.

It was argued that the damping force peak that occurs at the input (or driving) frequency is necessary for controlling the forced response of the system. The peaks at higher frequencies due to skyhook control, however, are not desirable - although always present - because they introduce corresponding peaks in the sprung mass acceleration that can cause ancillary vibration problems. For most systems (such as the seat system that was tested here) the high frequency peaks can significantly diminish any isolation benefits that are gained by the skyhook damper. When using skyhook dampers, such effects must be considered and their impact on the system dynamics carefully studied.

\section{References}

[1] M. Ahmadian and R.H. Marjoram, Effects of passive and semiactive suspensions on body and wheelhop control, Journal of Commercial Vehicles 98 (1989), 596-604.
[2] M. Ahmadian and R.H. Marjoram, On the development of a simulation model for tractor semitrailer systems with semiactive suspensions, in Proceedings of the Special Joint Symposium on Advanced Technologies, 1989 ASME Winter Annual Meeting, San Francisco, CA, DSC13, 1989.

[3] M. Ahmadian, On the Isolation Properties of Semiactive Dampers, Journal of Vibration and Control 5(2) (1999), 217232.

[4] J.D. Carlson and M.J. Chrzan, Magneto rheological Fluid Dampers, Patent 5 (1994), 277, 281.

[5] J.D. Carlson, D.M. Catanzarite and K.A. St. Clair, Commercial Magneto rheological Fluid Devices, International Conference On Electro-Rheological, Magneto rheological Suspensions and Associated Technology, Sheffield, 1995.

[6] R.M. Chalasani, Ride performance potential of active suspension systems-Part II: Comprehensive analysis based on a fullcar model, in Proceedings of the 1986 ASME Winter Annual Meeting, Los Angeles, CA, 1986.

[7] R.M. Chalasani, Ride Performance Potential of Active Suspension Systems-Part I: Simplified Analysis Based on a Quarter-Car Model, in Proceedings of the 1986 ASME Winter Annual Meeting, Los Angeles, CA, 1986.

[8] M.J. Crosby and D.C. Karnopp, The active damper, The Shock and Vibrations Bulletin 43, Naval Research Laboratory, Washington, DC, 1973.

[9] D.J. Inman, Engineering Vibration, Prentice-Hall, Englewood Cliffs, NJ, 1996.

[10] International Standards Organization, Earth-Moving Machinery - Laboratory Evaluation of Operator Seat Vibration, Standard 7096, 1994.

[11] D.E. Ivers and L.R. Miller, Semiactive suspension technology: An evolutionary view, ASME Advanced Automotive Technologies, DE-40, Book No. H00719-1991, 1991, pp. 327-346. 
[12] D.C. Karnopp and M.J. Cosby, System for controlling the transmission of energy between spaced members, US Patent $3,807,678,1974$

[13] E.J. Krasnicki, Comparison of analytical and experimental results for a semiactive vibration isolator, Shock and Vibration Bulletin 50 (1980).

[14] L.R. Miller, An approach to semiactive control of multipledegree-of-freedom systems, Ph.D. Thesis, North Carolina State University, Raleigh, NC, 1988.
[15] B.A. Reichert, Application of Magneto Rheological Dampers for Vehicle Seat Suspensions, Master of Science Thesis, Virginia Polytechnic University and State University, Blacksburg, Virginia, 1997.

[16] X. Song, Design of Adaptive Vibration Control Systems with Application to Magneto-Rheological Dampers, Doctor of Philosophy Dissertation, Virginia Polytechnic University and State University, Blacksburg, Virginia, 1999. 

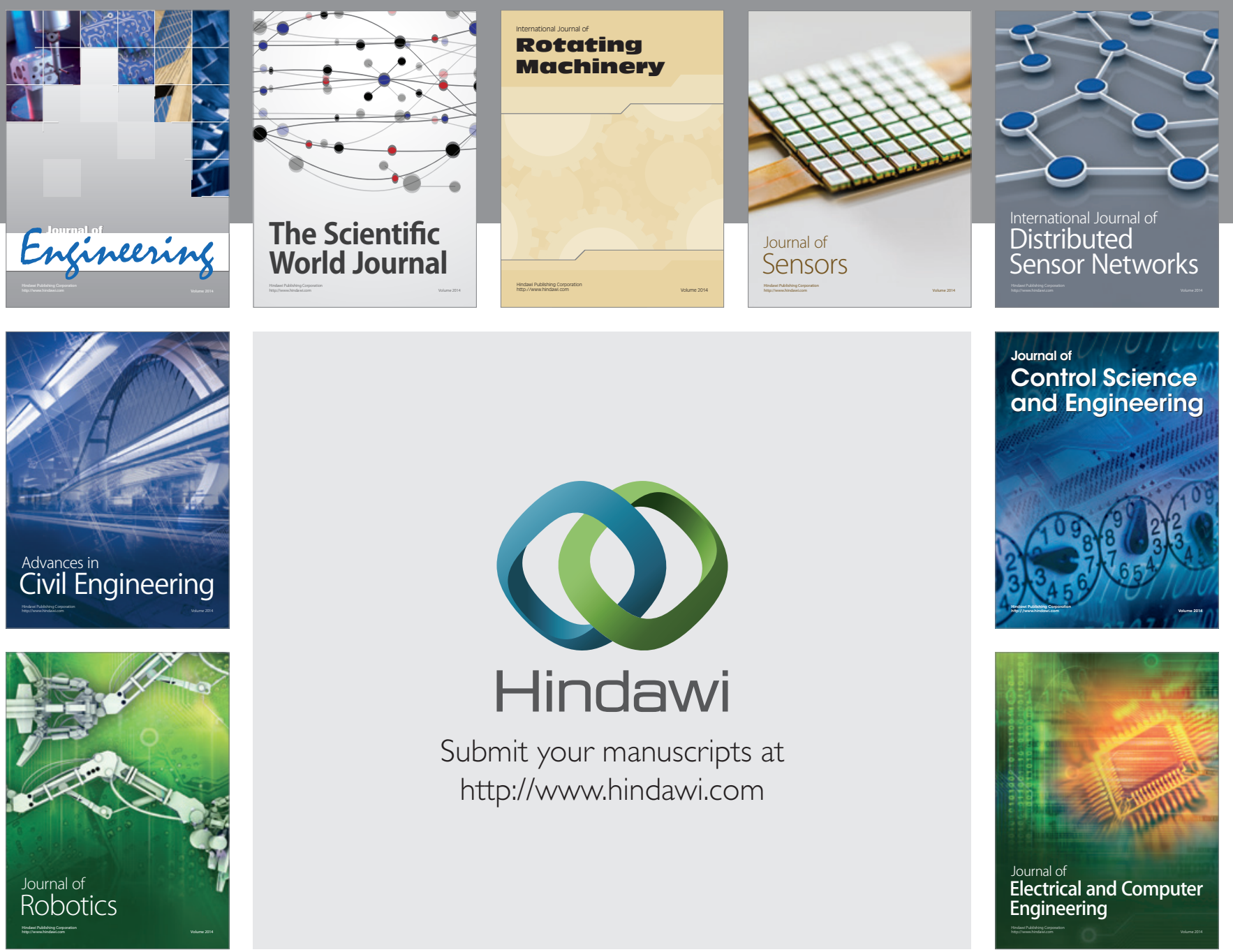

Submit your manuscripts at

http://www.hindawi.com
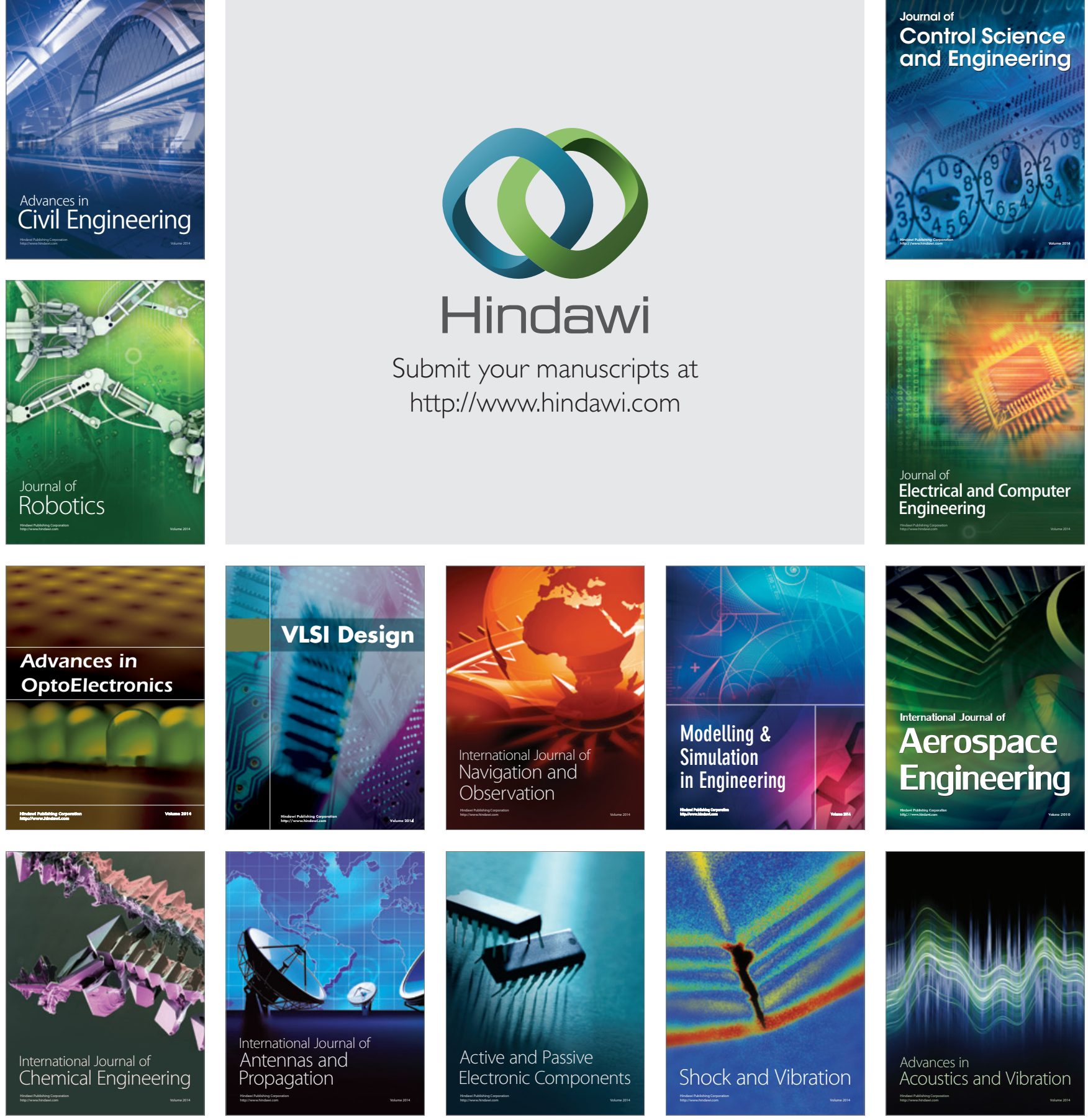